\title{
The human brain produces fructose from glucose
}

\author{
Janice J. Hwang, ${ }^{1}$ Lihong Jiang, ${ }^{2}$ Muhammad Hamza, ${ }^{1}$ Feng Dai, ${ }^{3}$ Renata Belfort-DeAguiar, ${ }^{1}$ \\ Gary Cline, ${ }^{1}$ Douglas L. Rothman, ${ }^{2}$ Graeme Mason, ${ }^{2}$ and Robert S. Sherwin ${ }^{1}$
}

${ }^{1}$ Section of Endocrinology and ${ }^{2}$ Department of Radiology \& Biomedical Imaging, Yale School of Medicine, New Haven, Connecticut, USA. ${ }^{3}$ Yale Center for Analytical Sciences, Yale School of Public Health, New Haven, Connecticut, USA.

Fructose has been implicated in the pathogenesis of obesity and type 2 diabetes. In contrast to glucose, CNS delivery of fructose in rodents promotes feeding behavior. However, because circulating plasma fructose levels are exceedingly low, it remains unclear to what extent fructose crosses the blood-brain barrier to exert CNS effects. To determine whether fructose can be endogenously generated from glucose via the polyol pathway (glucose $\rightarrow$ sorbitol $\rightarrow$ fructose) in human brain, 8 healthy subjects ( 4 women/4 men; age, $28.8 \pm 6.2$ years; BMI, $23.4 \pm 2.6$; HbA1C, $4.9 \% \pm 0.2 \%$ ) underwent ${ }^{1} \mathrm{H}$ magnetic resonance spectroscopy scanning to measure intracerebral glucose and fructose levels during a 4-hour hyperglycemic clamp (plasma glucose, $220 \mathrm{mg} / \mathrm{dl}$ ). Using mixed-effects regression model analysis, intracerebral glucose rose significantly over time and differed from baseline at $\mathbf{2 0}$ to $\mathbf{2 3 0}$ minutes. Intracerebral fructose levels also rose over time, differing from baseline at $\mathbf{3 0}$ to $\mathbf{2 3 0}$ minutes. The changes in intracerebral fructose were related to changes in intracerebral glucose but not to plasma fructose levels. Our findings suggest that the polyol pathway contributes to endogenous CNS production of fructose and that the effects of fructose in the CNS may extend beyond its direct dietary consumption.

Authorship note: J.J. Hwang and L. jiang contributed equally to this work.

Conflict of interest: The authors have declared that no conflict of interest exists.

Submitted: September 7, 2016 Accepted: January 6, 2017 Published: February 23, 2017

Reference information: JCI Insight. 2017;2(4):e90508. https:// doi.org/10.1172/jici.nsight.90508.

\section{Introduction}

Fructose has generated much attention as a potential driver of the US obesity and diabetes epidemics (1). In the brain, fructose, in contrast to glucose, appears to promote feeding behavior in rodents (2), and in humans, acute ingestion of fructose or glucose causes different patterns of cerebral blood flow changes in brain regions that influence eating behavior $(3,4)$. Because glucose enters the brain via passage across the blood-brain barrier $(\mathrm{BBB})(5,6)$, it is generally assumed that the CNS effects of fructose are mediated by peripheral fructose crossing the BBB as well (7). However, because of its efficient metabolism by the liver, extrahepatic circulating levels of fructose are exceedingly low. Thus, the extent to which peripheral fructose can cross the BBB to generate its CNS effects remains uncertain.

In this study, we use magnetic resonance spectroscopy (MRS) scanning, which allows for noninvasive direct measurements of metabolite concentrations to test an alternate mechanism, namely that fructose is produced endogenously within the human brain from glucose via the polyol pathway (glucose $\rightarrow$ sorbitol $\rightarrow$ fructose). The polyol pathway is an accessory glucose pathway that bypasses glycolysis $(8,9)$, and in the setting of hyperglycemia, up to $30 \%$ of glucose can be metabolized via this alternate pathway $(10,11)$. The polyol pathway is present throughout the human body, including the brain $(12,13)$, and it is responsible for the 20-fold higher levels of fructose observed in cerebrospinal fluid compared with blood (14).

In addition to fructose generation, flux through the polyol pathway also generates oxidative stress via production of $\mathrm{NADH}$ as well as because aldose reductase competes with glutathione reductase for NADPH, resulting in decreased glutathione (15). It is noteworthy that, in the periphery, this excessive oxidative stress has been implicated in the development of peripheral neuropathy (16), diabetic retinopathy and cataracts (17-20), nephropathy (21), fatty liver (22), and macrovascular disease (23) as well as platelet dysfunction $(24,25)$. In the brain, however, its role remains unclear. Thus, the current proof-of-concept study examines whether peripheral hyperglycemia can drive intracerebral production of fructose via the polyol pathway in lean, healthy individuals. 

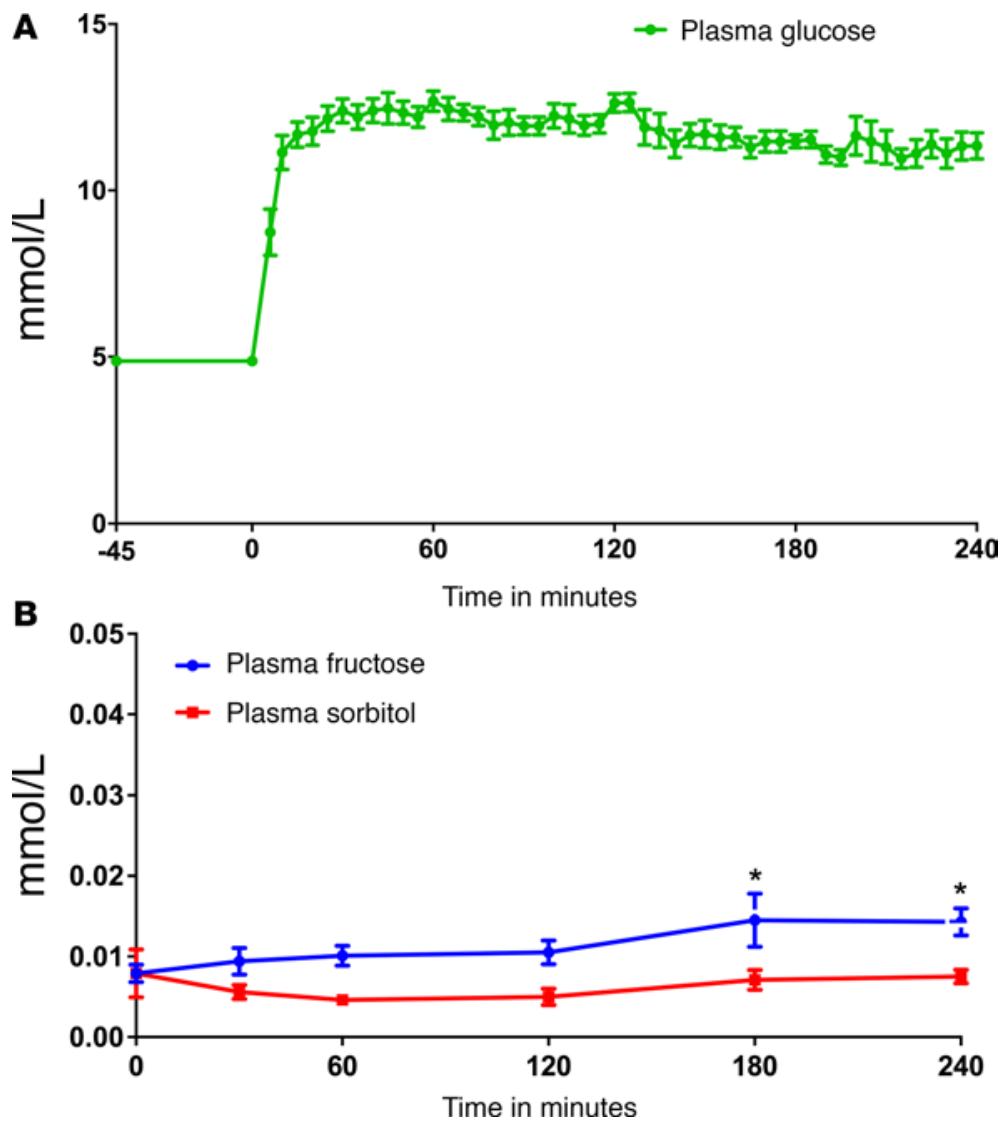

Figure 1. (A) Plasma glucose levels over time. (B) Plasma fructose and sorbitol levels over time. ${ }^{*} P<0.0001$ at times 180 and 240 minutes compared with baseline for fructose. Data represent mean \pm SEM.

\section{Results}

Baseline fasting plasma glucose was $4.87 \pm 0.15 \mathrm{mmol} / 1$ and rapidly increased to approximately $12 \mathrm{mmol} / 1$ during the hyperglycemic clamp $(P<0.0001)$ (Figure 1A). Fasting levels of plasma fructose and sorbitol were much lower at baseline $(0.008 \pm 0.001 \mathrm{mmol} / 1$ and $0.008 \pm 0.003$ mmol/1, respectively), and plasma fructose levels rose slightly (peak $0.0145 \mathrm{mmol} / 1$ at $180 \mathrm{~min}$ ) (Figure 1B). No differences in plasma sorbitol levels were detected throughout (Figure 1B).

Baseline spectra were subtracted from spectra obtained every 10 minutes during the course of the glucose infusion in order to generate the difference spectra (Figure 2A), which were then compared with the reference spectra for glucose, fructose, and glucose and fructose obtained under identical scanning parameters (Figure 2B). Compared with baseline, intracerebral glucose levels rose significantly over time, beginning at 10 minutes $(P=$ $0.0004)$ through the end of the study $(P<0.0001)$. Moreover, brain fructose levels also rose significantly compared with baseline, beginning at 20 minutes $(P=0.007)$ and extending through the end of the study $(P<0.01)$ (Figure $3 \mathrm{~A})$. Each of the 8 subjects had increases in intracerebral glucose (Figure 3B) and intracerebral fructose (Figure 3C). The magnitude of the change in intracerebral fructose concentration (obtained from times $60-180$ minutes) was about $30 \%-35 \%$ of the change in intracerebral glucose (Figure 3A). Furthermore, the change in brain fructose levels was associated with the change in brain glucose levels $(\beta$ coefficient $=0.18$, SEM 0.03, $P<0.0001)$ but not plasma fructose levels $(P=0.36)$.

\section{Discussion}

The current data demonstrate that fructose is rapidly generated in the human brain during hyperglycemia. Our findings are consistent with those of a prior study in rats using ${ }^{19} \mathrm{~F}-\mathrm{MRS}$, which showed that, following i.v. 3-fluoro-3-deoxy-D-glucose infusion, there was a rise in intracerebral 3-fluoro-3-deoxy-D-sorbitol and 3-fluoro-3-deoxy-D-fructose. Furthermore, the production of sorbitol and fructose was blocked by sorbinil, an inhibitor of the rate-limiting enzyme, aldose reductase (26). Together with the previous finding that fructose and sorbitol are present in human cerebrospinal fluid at markedly higher concentrations than in the periphery $(12,14,27)$, the present observations suggest that endogenous human brain fructose production may be a key underappreciated factor modulating ingested sugar's effect on the brain. While it is possible that plasma fructose may cross the BBB, we observed that the rise of intracerebral fructose occurred much earlier (by 20 minutes) than the modest increase in peripheral fructose levels (at 180 minutes). Furthermore, in rats, a fructose infusion raising plasma fructose nearly 140 -fold only increased brain fructose concentrations by 2 -fold (3). Taken together, it is highly unlikely that plasma fructose crossing into the brain significantly contributed to the rising brain fructose levels.

In the present study, the change in brain fructose was about $30 \%$ of the change in brain glucose $(\sim 0.5$ $\mathrm{mmol} / 1$ for fructose, $1.5 \mathrm{mmol} / 1$ for glucose). When considered with our recent finding that plasma fructose levels are only $0.1 \%$ of plasma glucose levels, compared with CSF in which fructose is $6 \%-7 \%$ of glucose (28), these findings suggest that relative changes in brain fructose may be greater than relative changes in brain glucose levels, potentially making fructose a highly sensitive neuronal signal for carbohydrate ingestion. Interestingly, in Drosophila, any nutritious carbohydrate ingestion results in increased hemolymph fructose levels (through the polyol pathway), which in turn acts on a nutrient sensor in the fruit fly 
A

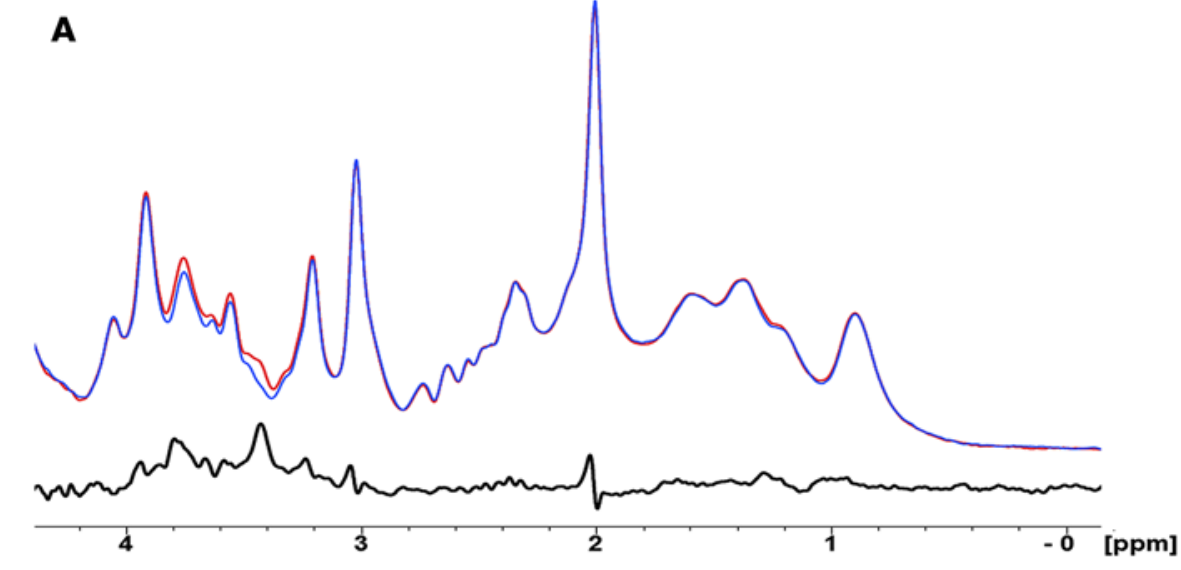

B
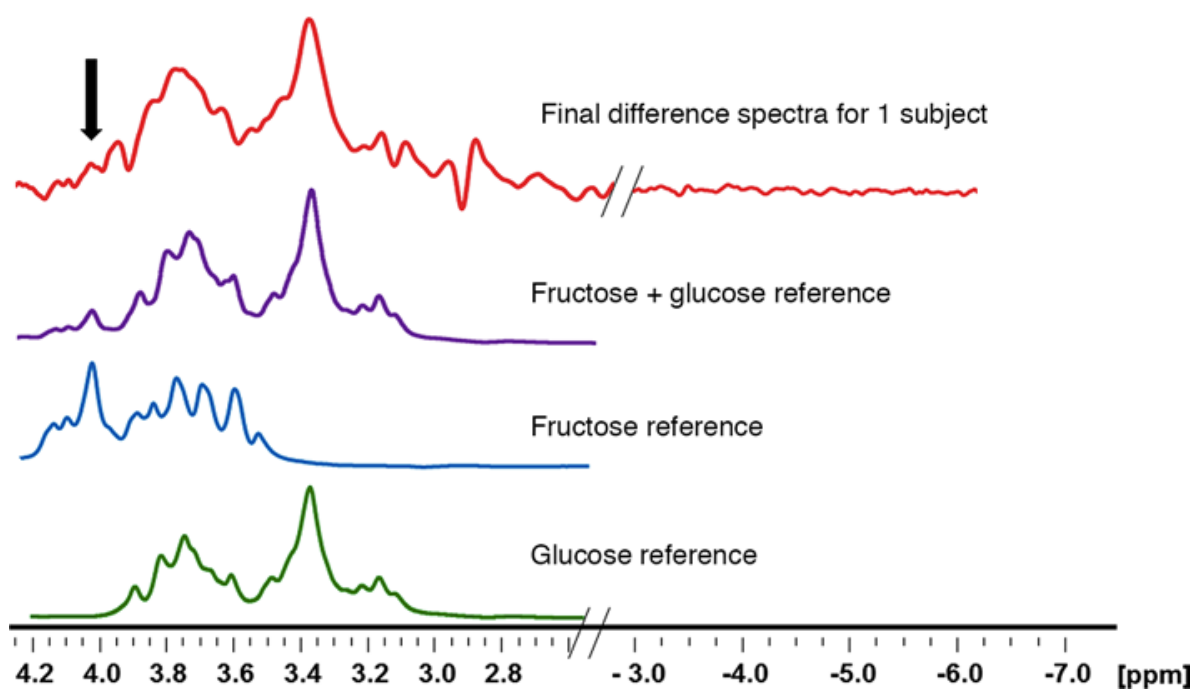

Figure 2. Intracerebral fructose and glucose levels. (A) Difference spectrum procedure showing for data from 1 subject. Blue spectra were obtained at baseline; red spectra were obtained at 10 minutes; black spectra is the difference between the red and blue spectra. (B) In vivo difference spectrum (final difference spectra) from 1 subject compared with reference spectra for fructose plus glucose, pure fructose, and pure glucose obtained under in vivo conditions of temperature $37^{\circ} \mathrm{C}, \mathrm{pH} 7.4$, and ionic strength of $150 \mathrm{mM}$. The fructose and glucose solution spectra were added, with their relative intensities adjusted to match their relative concentrations measured in vivo. The spectral regions plotted from the subject have been extended to include the region from -3 to $-7 \mathrm{ppm}$ where only noise is present in order to show the signal-to-noise ratio of the fructose peak. The arrow denotes the unique fructose peak at 4.0-4.1 ppm used to distinguish fructose from glucose.

brain (29). Whether fructose plays a similar role in modulating nutrient sensing in the mammalian or human brain requires further investigation.

This proof-of-concept study has some limitations. First, using ${ }^{1} \mathrm{H}$ MRS scanning, we were only able to measure changes in concentration and not absolute concentrations of fructose in the human brain. In addition, because the chemical spectrum for sorbitol overlaps completely with the spectra for glucose and fructose, we were unable to determine changes in sorbitol levels, which may have contributed to the difference spectrum intensity. Furthermore, in order to maximize sensitivity of signal detection, we scanned the occipital lobe and thus were not able to determine whether known regional variability in glucose utilization translates to differences in polyol pathway activity. Finally, our study design assumes that insulin does not regulate the polyol pathway. While animal studies have suggested that the polyol pathway activity is purely substrate dependent and not insulin dependent $(30,31)$, we did not directly control for the effects of insulin (or other hormones and metabolites, such as lactate; ref. 32) that conceivably might have indirect effects on the polyol pathway.

In light of the current obesity and diabetes epidemics, dietary fructose has garnered attention as a potential driver of these epidemics in part because of the high prevalence of high-fructose sweeteners (typically fructose/glucose ratios are 55:45 to 60:40) in the US diet. However, fructose is almost never consumed in isolation, and our finding that glucose levels drive brain fructose levels suggests that any sugar consumption that raises circulating glucose may in large part mediate fructose's effects in the brain. Why the human brain produces fructose at such high concentrations compared with the plasma remains unclear. In humans, Glut 5 , the principal fructose transporter (33), is present predominantly on microglial cells $(34,35)$, raising the possibility that endogenous fructose production in the brain may alter neuronal and glial interactions.

A growing body of evidence suggests that chronic hyperglycemia leads to many adverse effects on brain function, particularly neurovascular disorders and cognitive impairment $(36,37)$. However, the underlying mechanisms behind these associations remain unclear. The current data demonstrating that fructose is produced in the human brain in response to hyperglycemia via the polyol pathway activity as well as evidence for the presence of aldose reductase, the rate-limiting enzyme in the polyol pathway, throughout the 

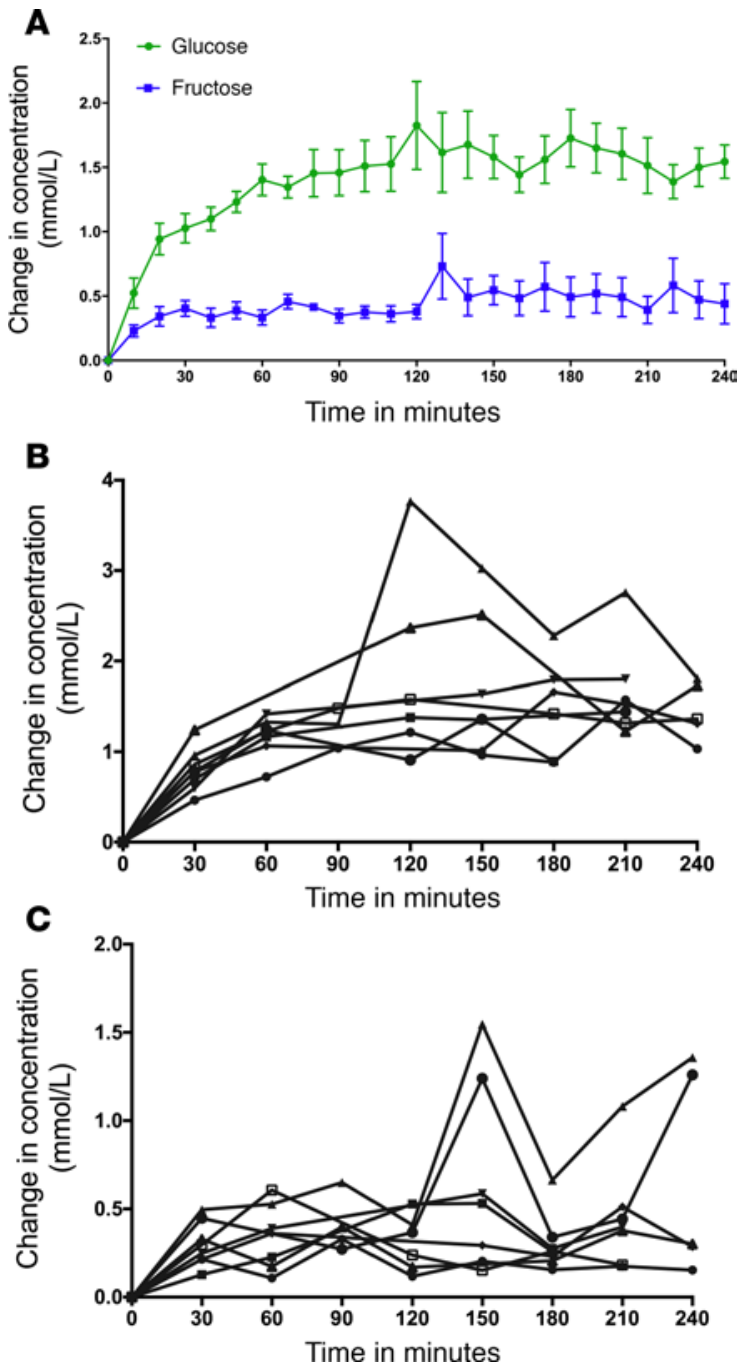

Figure 3. Time course of the changes in glucose and fructose concentration during the glucose infusion. (A) Change in intracerebral glucose was different compared with baseline beginning at 10 minutes $(P<0.001)$. The change in fructose was different compared with baseline beginning at 20 minutes $(P<0.01$ from time 20 minutes to 120 minutes, $P<0.0001$ from time 130 minutes to 220 minutes). Data expressed as mean \pm SEM. (B) Change in intracerebral glucose plotted for each individual subject. (C) Change in intracerebral fructose plotted for each individual subject. The vertical scale is multiplied by $2 \times$ relative to the individual glucose difference plot.

human brain, particularly in the cerebral cortex, basal ganglia, and hippocampus (11), may have particular implications for disordered eating behavior and disordered cognition in patients with diabetes.

\section{Methods}

Participants. Eight healthy participants with normal weight were recruited with flyers and advertisements from the greater New Haven area (Table 1). Exclusion criteria included contraindications to entering an MRI, medication use (other than contraceptives for women), smoking history, illicit drug, or recent steroid use. Women who were breastfeeding, seeking pregnancy, or shown to be pregnant by urine test were also excluded.

Experimental protocol. Participants arrived at 7 AM following an overnight fast. One i.v. catheter was inserted into a distal arm for blood sampling. A second i.v. was inserted into the contralateral arm for $20 \%$ dextrose infusion that was adjusted every 5-10 minutes to achieve and maintain plasma glucose at $12 \mathrm{mmol} / 1$. Additional blood samples were obtained for measurement of plasma sorbitol and fructose at baseline and 30, 60, 120, 180, and 240 minutes during hyperglycemia. The primary endpoint was change in intracerebral glucose and fructose levels. Secondary endpoints included changes in systemic sorbitol and fructose levels.

${ }^{1} H$ MRS scanning. Participants were positioned supine in a $4.0 \mathrm{~T}$ wholebody magnet interfaced to a Bruker AVANCE spectrometer (Bruker Instruments), with the head immobilized with foam inserts on top of a radiofrequency probe with a ${ }^{1} \mathrm{H}$ circular coil as previously described $(6,38)$. After tuning, calibration, and acquisition of scout images for anatomical localization, intracerebral glucose and fructose concentration signals were obtained using stimulated echo acquisition mode localization (39) in $30 \times 20 \times 30 \mathrm{~mm}^{3}$ voxels in the occipital lobe for 20 minutes at baseline and then every 10 minutes over 4 hours of hyperglycemia. The sequence parameters were TR =2,000 ms; TE/ $\mathrm{TM}=15 \mathrm{~ms} / 10 \mathrm{~ms}$; bandwidth $=5,000 \mathrm{~Hz}$; sampling points $=2,048$. Spectra were acquired with B0-lock and retrospective frequency adjustment for motion correction.

Although there is some overlap in their chemical spectra, fructose has unique peaks located at 4.0-4.1 ppm, which were used to distinguish it from glucose (Human Metabolome Database v3.6; refs. 40, 41). Baseline spectra were subtracted to eliminate overlap from other brain metabolites not directly affected by glucose levels. Changes in metabolite levels were measured by peak integration referenced to total voxel water signal and creatine, in the nondifferenced spectra, to determine concentration. Glucose integrals were obtained by integrating the large peak from 3.32 to 3.54 ppm and scaled back total intensity from 3.10 to $3.96 \mathrm{ppm}$ in the free glucose spectrum (1:2.9), which contains 11 protons. Fructose integrals were obtained by integrating the peak from 3.96 to $4.07 \mathrm{ppm}$ and scaled back total intensity from 3.54 to $4.20 \mathrm{ppm}$ from the free fructose spectrum (1:4.8), which contains 12 protons. The concentrations of glucose and fructose were then compared with the creatine $\mathrm{CH} 3$ peak in the in vivo spectrum, which was assumed to be $10 \mathrm{mmol} / \mathrm{kg}$ and which contains 3 protons. Reference solutions of glucose (100 mM), sorbitol (100 mM), and fructose $(100 \mathrm{mM})$ were

\section{Table 1. Participant characteristics}

\begin{tabular}{|cc|}
\hline Characteristic & Mean (SD) \\
\hline Age (yr) & $28.8(6.2)$ \\
\hline Sex (male/female) & $4 / 4$ \\
\hline BMI $\left(\mathrm{kg} / \mathrm{m}^{2}\right)$ & $23.4(2.6)$ \\
\hline HbA1C $(\%)$ & $4.9(0.2)$ \\
\hline
\end{tabular}


prepared in $100 \mathrm{mM}$ phosphate buffer ( $\mathrm{pH}$ 7.4) and measured after preheating the reference solutions to $37^{\circ} \mathrm{C}$. Spectra were acquired using the same pulse sequence and parameters as used in vivo. Sorbitol peaks overlapped with glucose and fructose. While the peak pattern in the difference spectra was most consistent with glucose and fructose, we cannot rule out some degree of sorbitol contribution.

Laboratory analysis. Plasma glucose levels were measured via glucose oxidase (YSI Inc.). Plasma sorbitol and fructose were measured using gas chromatography-tandem mass spectrometry.

Statistics. The analyses of every repeatedly measured variable, including intracerebral glucose and fructose and plasma metabolite levels, were performed using the mixed-effects regression model method, taking in account within-subject correlation of repeated measures using a prespecified compound symmetry covariance matrix. Age, gender, and BMI were adjusted as covariates (i.e., as fixed effects). The association of intracerebral fructose with intracerebral glucose levels was also modeled using the mixed-effects regression model, in which the glucose level was included as a covariate in addition to age, gender, and BMI. The association of intracerebral fructose level with plasma fructose level was modeled in a similar manner. All the analyses were performed using SAS, version 9.4. A 2-sided $P$ value of less than 0.05 was considered to be statistically significant.

Study approval. The Yale University Human Investigation Committee (New Haven, Connecticut, USA) approved the protocol, and all participants provided written informed consent prior to study participation.

\section{Author contributions}

$\mathrm{JJH}, \mathrm{LJ}$, and RSS had full access to all of the data in the study and take responsibility for the integrity of the data and the accuracy of the data analysis. JJH, LJ, DLR, GM, and RSS conceived of and designed the study. JJH, LJ, MH, RBD, and GC acquired data. JJH, LJ, MH, FD, RBD, DLR, GM, and RSS analyzed and interpreted data. JJH, LJ, MH, RBD, GC, DLR, GM, and RSS wrote the manuscript. JJH, LJ, and FD performed statistical analysis.

\section{Acknowledgments}

We gratefully acknowledge the help of the Yale Hospital Research Unit nurses and staff and the Yale Core lab staff as well as the staff at the Yale Magnetic Resonance Center. This study was supported in part by grants from the NIH (R01DK020495 to RSS, KL2TR000140 to JJH, K23 DK109284 to JJH, P30 DK045735 to RSS, R01AA021984 to GM, R01NS087568 to DLR, and SIG 11S10OD010613-01); the Yale Center for Clinical Investigation, supported by the Clinical Translational Science Award (UL1 RR024139); the Endocrine Fellows Foundation (to JJH); the Yale Diabetes Center Pilot award (P30DK045735 to JJH); and the Mouse Metabolic Phenotyping Center (U2C DK-059635).

Address correspondence to: Robert Sherwin or Janice Hwang, The Anlyan Center, TAC 141S, PO Box 208020, New Haven, Connecticut 06520, USA. Phone: 203.785.6222; Email: Robert.Sherwin@yale.edu (R.S. Sherwin); Janice.Hwang@yale.edu (J.J. Hwang).

1. Stanhope KL, Schwarz JM, Havel PJ. Adverse metabolic effects of dietary fructose: results from the recent epidemiological, clinical, and mechanistic studies. Curr Opin Lipidol. 2013;24(3):198-206.

2. Cha SH, Wolfgang M, Tokutake Y, Chohnan S, Lane MD. Differential effects of central fructose and glucose on hypothalamic malonyl-CoA and food intake. Proc Natl Acad Sci USA. 2008;105(44):16871-16875

3. Page KA, et al. Effects of fructose vs glucose on regional cerebral blood flow in brain regions involved with appetite and reward pathways. JAMA. 2013;309(1):63-70

4. Luo S, Monterosso JR, Sarpelleh K, Page KA. Differential effects of fructose versus glucose on brain and appetitive responses to food cues and decisions for food rewards. Proc Natl Acad Sci USA. 2015;112(20):6509-6514.

5. Gruetter R, et al. Direct measurement of brain glucose concentrations in humans by 13C NMR spectroscopy. Proc Natl Acad Sci USA. 1992;89(3):1109-1112.

6. Gruetter R, Novotny EJ, Boulware SD, Rothman DL, Shulman RG. 1H NMR studies of glucose transport in the human brain. J Cereb Blood Flow Metab. 1996;16(3):427-438.

7. Wuest M, et al. Radiopharmacological evaluation of 6-deoxy-6-[18F]fluoro-D-fructose as a radiotracer for PET imaging of GLUT5 in breast cancer. Nucl Med Biol. 2011;38(4):461-475.

8. Jeffery J, Jörnvall H. Enzyme relationships in a sorbitol pathway that bypasses glycolysis and pentose phosphates in glucose metabolism. Proc Natl Acad Sci USA. 1983;80(4):901-905.

9. Das B, Srivastava SK. Activation of aldose reductase from human tissues. Diabetes. 1985;34(11):1145-1151.

10. Bhatnagar A, Srivastava SK. Aldose reductase: congenial and injurious profiles of an enigmatic enzyme. Biochem Med Metab Biol. 1992;48(2):91-121. 
11. Chung SS, Ho EC, Lam KS, Chung SK. Contribution of polyol pathway to diabetes-induced oxidative stress. J Am Soc Nephrol. 2003;14(8 Suppl 3):S233-S236.

12. Gabbay KH. The sorbitol pathway and the complications of diabetes. N Engl J Med. 1973;288(16):831-836

13. Picklo MJ, Olson SJ, Markesbery WR, Montine TJ. Expression and activities of aldo-keto oxidoreductases in Alzheimer disease. J Neuropathol Exp Neurol. 2001;60(7):686-695.

14. Hwang JJ, et al. Fructose levels are markedly elevated in cerebrospinal fluid compared to plasma in pregnant women. PLoS One. 2015;10(6):e0128582.

15. Tang WH, Martin KA, Hwa J. Aldose reductase, oxidative stress, and diabetic mellitus. Front Pharmacol. 2012;3:87.

16. Greene DA, Lattimer SA, Sima AA. Sorbitol, phosphoinositides, and sodium-potassium-ATPase in the pathogenesis of diabetic complications. N Engl J Med. 1987;316(10):599-606.

17. Nishimura C, Saito T, Ito T, Omori Y, Tanimoto T. High levels of erythrocyte aldose reductase and diabetic retinopathy in NIDDM patients. Diabetologia. 1994;37(3):328-330.

18. Nishimura C, et al. The level of erythrocyte aldose reductase is associated with the severity of diabetic retinopathy. Diabetes Res Clin Pract. 1997;37(3):173-177.

19. Ito T, Nishimura C, Takahashi Y, Saito T, Omori Y. The level of erythrocyte aldose reductase: a risk factor for diabetic neuropathy? Diabetes Res Clin Pract. 1997;36(3):161-167.

20. Lee AY, Chung SS. Contributions of polyol pathway to oxidative stress in diabetic cataract. FASEB J. 1999;13(1):23-30.

21. Lanaspa MA, et al. Endogenous fructose production and fructokinase activation mediate renal injury in diabetic nephropathy J Am Soc Nephrol. 2014;25(11):2526-2538.

22. Lanaspa MA, et al. Endogenous fructose production and metabolism in the liver contributes to the development of metabolic syndrome. Nat Commun. 2013;4:2434.

23. Vedantham S, et al. Aldose reductase drives hyperacetylation of Egr-1 in hyperglycemia and consequent upregulation of proinflammatory and prothrombotic signals. Diabetes. 2014;63(2):761-774.

24. Tang WH, et al. Glucose and collagen regulate human platelet activity through aldose reductase induction of thromboxane. J Clin Invest. 2011;121(11):4462-4476.

25. Tang WH, et al. Aldose reductase-mediated phosphorylation of p53 leads to mitochondrial dysfunction and damage in diabetic platelets. Circulation. 2014;129(15):1598-1609.

26. Nakada T, Kwee IL, Conboy CB. Noninvasive in vivo demonstration of 2-fluoro-2-deoxy-D-glucose metabolism beyond the hexokinase reaction in rat brain by 19F nuclear magnetic resonance spectroscopy. J Neurochem. 1986;46(1):198-201.

27. Hubbard RSaRNM The fructose content of spinal fluid. J Biol Chem. 1937; 119: 647-661.

28. Hwang JJ, et al. Fructose levels are markedly elevated in cerebrospinal fluid compared to plasma in pregnant women. PLoS ONE. 2015;10(6):e0128582.

29. Miyamoto T, Slone J, Song X, Amrein H. A fructose receptor functions as a nutrient sensor in the Drosophila brain. Cell. 2012;151(5):1113-1125.

30. Spinowitz BS, Altszuler N. Increased production of fructose by the brain in dogs made diabetic by alloxan and by growth hormone. Diabetes. 1973;22(11):820-824.

31. Margolis RU, Press R, Altszuler N. Increased fructose production by the brain in alloxan diabetes. Brain Res. 1972;38(2):371-375.

32. Adams SH, Stanhope KL, Grant RW, Cummings BP, Havel PJ. Metabolic and endocrine profiles in response to systemic infusion of fructose and glucose in rhesus macaques. Endocrinology. 2008;149(6):3002-3008.

33. Mueckler M, Thorens B. The SLC2 (GLUT) family of membrane transporters. Mol Aspects Med. 2013;34(2-3):121-138

34. Izumi Y, Zorumski CF. Glial-neuronal interactions underlying fructose utilization in rat hippocampal slices. Neuroscience. 2009;161(3):847-854.

35. Horikoshi Y, et al. Human GLUT5 immunolabeling is useful for evaluating microglial status in neuropathological study using paraffin sections. Acta Neuropathol. 2003;105(2):157-162.

36. Strachan MW, Reynolds RM, Marioni RE, Price JF. Cognitive function, dementia and type 2 diabetes mellitus in the elderly. Nat Rev Endocrinol. 2011;7(2):108-114.

37. Cukierman T, Gerstein HC, Williamson JD. Cognitive decline and dementia in diabetes--systematic overview of prospective observational studies. Diabetologia. 2005;48(12):2460-2469.

38. Gruetter R, Rothman DL, Novotny EJ, Shulman GI, Prichard JW, Shulman RG. Detection and assignment of the glucose signal in 1H NMR difference spectra of the human brain. Magn Reson Med. 1992;27(1):183-188.

39. Thompson RB, Allen PS. Response of metabolites with coupled spins to the STEAM sequence. Magn Reson Med. 2001;45(6):955-965.

40. Wishart DS, et al. HMDB 3.0--The Human Metabolome Database in 2013. Nucleic Acids Res. 2013;41(Database issue):D801-D807.

41. Wishart DS, et al. HMDB: the Human Metabolome Database. Nucleic Acids Res. 2007;35(Database issue):D521-D526. 